\title{
Flow topologies in different regimes of premixed turbulent combustion: A direct numerical simulation analysis
}

\author{
Daniel H. Wacks, ${ }^{1, *}$ Nilanjan Chakraborty,,${ }^{1, \dagger}$ Markus Klein,,${ }^{2,}$ \\ Paul G. Arias, ${ }^{3, \#}$ and Hong G. $\operatorname{Im}^{4, \pi}$ \\ ${ }^{1}$ School of Mechanical and Systems Engineering, University of Newcastle, Claremont Road, \\ Newcastle NE1 7RU, United Kingdom \\ ${ }^{2}$ Fakultät für Luft- und Raumfahrttechnik, Universität der Bundeswehr München, \\ Werner-Heisenberg-Weg 39, 85577 Neubiberg, Germany \\ ${ }^{3}$ Department of Mechanical Engineering, University of Michigan, Ann Arbor, Michigan 48109-2125, USA \\ ${ }^{4}$ Clean Combustion Research Center, King Abdullah University of Science and Technology, \\ Thuwal 23955-6900, Saudi Arabia
}

(Received 1 June 2016; published 2 December 2016)

\begin{abstract}
The distributions of flow topologies within the flames representing the corrugated flamelets, thin reaction zones, and broken reaction zone regimes of premixed turbulent combustion are investigated using direct numerical simulation data of statistically planar turbulent $\mathrm{H}_{2}$-air flames with an equivalence ratio $\phi=0.7$. It was found that the diminishing influence of dilatation rate with increasing Karlovitz number has significant influences on the statistical behaviors of the first, second, and third invariants (i.e., $P, Q$, and $R$ ) of the velocity gradient tensor. These differences are reflected in the distributions of the flow topologies within the flames considered in this analysis. This has important consequences for those topologies that make dominant contributions to the scalar-turbulence interaction and vortexstretching terms in the scalar dissipation rate and enstrophy transport equations, respectively. Detailed physical explanations are provided for the observed regime dependences of the flow topologies and their implications on the scalar dissipation rate and enstrophy transport.
\end{abstract}

DOI: 10.1103/PhysRevFluids.1.083401

\section{INTRODUCTION}

Turbulent flow fields often exhibit organized flow topologies in spite of their apparent chaotic nature. Perry and Chong [1] and Chong et al. [2] assigned all possible local small-scale three-dimensional flow topologies to eight categories based on the invariants $P, Q$, and $R$ of the velocity-gradient tensor $A_{i j}=\partial u_{i} / \partial x_{j}$, where $u_{i}$ is the $i$ th component of velocity vector. The topologies, denoted by $S 1-S 8$, distinguish eight regions in the three-dimensional $P-Q-R$ phase space, as described in Fig. 1. Several previous studies [3-8] analyzed the flow properties in the $Q-R$ plane for incompressible fluids (i.e., $P=-\nabla \cdot \vec{u}=0$ ). For compressible flows $(P \neq 0)$, however, one needs to account for $P-Q-R$ space [9-12]. The analyses of topologies in nonreacting compressible flow turbulence have indicated that the unstable node-saddle-saddle and stable focal-stretching topologies in the $Q-R$ plane dominate over other topologies.

In comparison to the large body of literature on local flow topologies in nonreacting turbulent flows, relatively little attention has been paid to their analysis in turbulent reacting flows [13-16].

\footnotetext{
*daniel.wacks@ncl.ac.uk

†Corresponding author: nilanjan.chakraborty@ncl.ac.uk

${ }^{\ddagger}$ markus.klein@unibw.de

\#pgarias@umich.edu

Ihong.im@kaust.edu.sa
} 

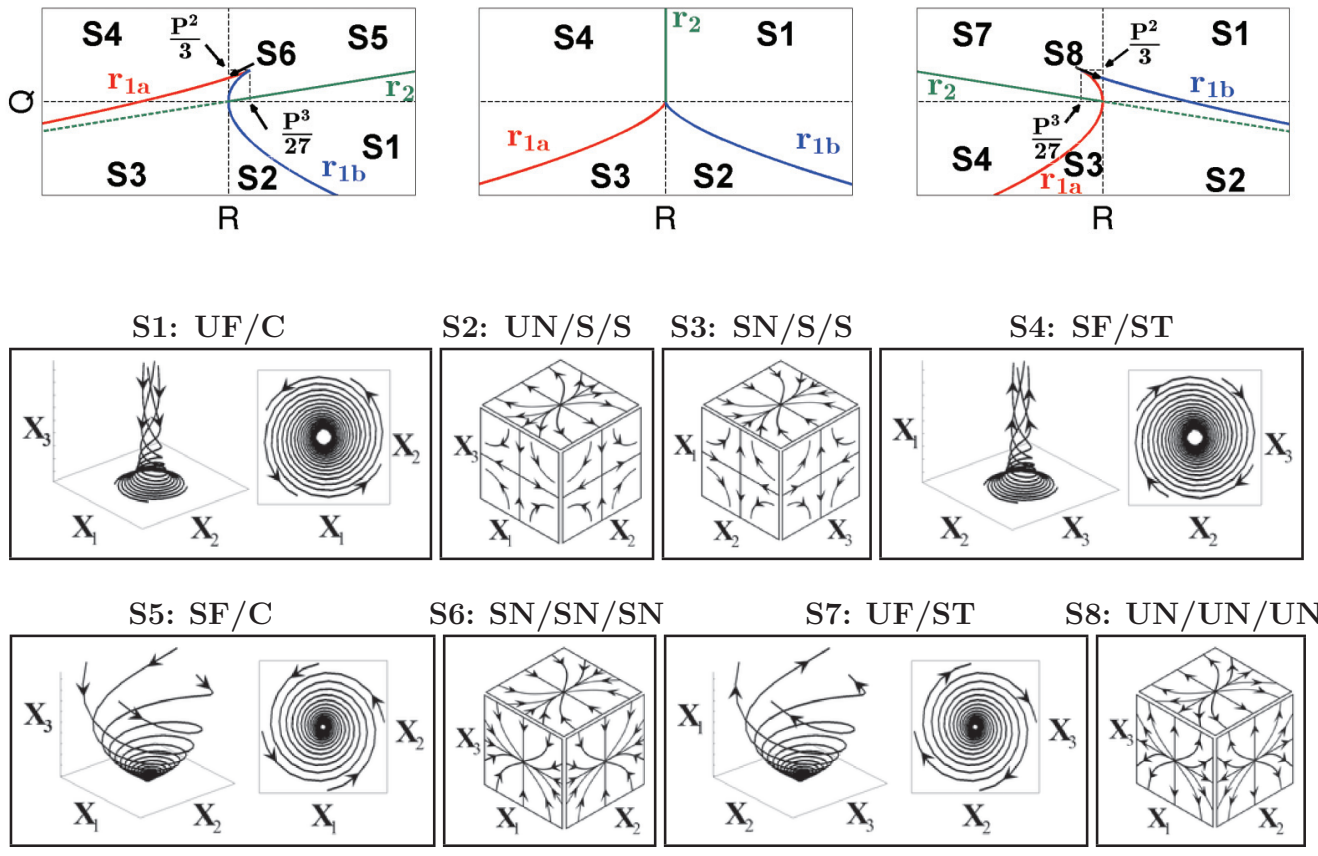

FIG. 1. Shown on top is the classification of $S 1-S 8$ topologies in the $Q-R$ plane for (left to right) $P>0$, $P=0$, and $P<0$. The lines $r_{1 a}$ (red), $r_{1 b}$ (blue), and $r_{2}$ (green) dividing the topologies are shown. Black dashed lines correspond to $Q=R=0$. The bottom shows the classification of $S 1-S 8$ topologies: UF, unstable focus; UN, unstable node; SF, stable focus; SN, stable node; S, saddle; C, compressing; and ST, stretching.

Tanahashi et al. [13] used $Q$ to distinguish strain-dominated and vorticity-dominated regions in a premixed flame and concluded that the vorticity vector remains perpendicular to the flame normal vector and that small-scale turbulence can survive even beyond the flame front. Grout et al. [14] analyzed the local flow topology of a nonpremixed jet in crossflow and reported that the highest heat release rates of the flame are associated with the regions with $S 8$ topology. Recently, Cifuentes et al. $[15,16]$ analyzed the topology distribution in a premixed turbulent flame based on a simple chemistry direct numerical simulation (DNS) database representing the flamelet combustion and demonstrated that the probability of finding focal (i.e., vortical) topologies decreases from the unburned gas side to the burned gas side. However, the differences in flow topology distribution within the flame for different regimes of premixed turbulent combustion are yet to be analyzed in the existing literature. These differences have important consequences on the scalar-turbulence interaction and vortex-stretching terms in the scalar dissipation rate and enstrophy transport equations, respectively $[17,18]$. Thus, the main objectives of this paper are (a) to identify the differences in the distribution of flow topologies in turbulent premixed flames representing different regimes of combustion and (b) to indicate the implications of the differences in topology distributions on the scalar-turbulence interaction and vortex-stretching terms.

A three-dimensional DNS database of statistically planar turbulent premixed $\mathrm{H}_{2}$-air flames with equivalence ratio $\phi=0.7$ [19] has been considered spanning different regimes of premixed combustion. The rest of the paper will be organized as follows. The mathematical background and numerical implementation pertaining to the current analysis will be presented in the next section. This will be followed by presentation of results and their subsequent discussion. Finally, the main findings will be summarized and conclusions will be drawn in the final section of this paper. 


\section{MATHEMATICAL BACKGROUND AND NUMERICAL IMPLEMENTATION}

Premixed combustion is often characterized using the nondimensional temperature $c_{T}=$ $\left(T-T_{0}\right) /\left(T_{a d}-T_{0}\right)$, which increases from zero to unity from unburned to burned gases, where $T, T_{0}$, and $T_{a d}$ are the dimensional, unburned gas, and adiabatic flame temperature, respectively. The local flow topologies can be characterized by the invariants of the velocity-gradient tensor $[1,2] A_{i j}=$ $\partial u_{i} / \partial x_{j}=S_{i j}+W_{i j}$, where $S_{i j}=0.5\left(A_{i j}+A_{j i}\right)$ and $W_{i j}=0.5\left(A_{i j}-A_{j i}\right)$ are the symmetric and antisymmetric components, respectively. Three eigenvalues $\lambda_{1}, \lambda_{2}$, and $\lambda_{3}$ of $A_{i j}$ are the solutions of the characteristic equation $\lambda^{3}+P \lambda^{2}+Q \lambda+R=0$, where $P, Q$, and $R$ are the invariants of $A_{i j}[1,2]$ :

$$
\begin{aligned}
& P=-\left(\lambda_{1}+\lambda_{2}+\lambda_{3}\right), \quad Q=0.5\left(P^{2}-S_{i j} S_{i j}+W_{i j} W_{i j}\right), \\
& R=\left(-P^{3}+3 P Q-S_{i j} S_{j k} S_{k i}-3 W_{i j} W_{j k} S_{k i}\right) / 3 .
\end{aligned}
$$

The discriminant $D=\left[27 R^{2}+\left(4 P^{3}-18 P Q\right) R+4 Q^{3}-P^{2} Q^{2}\right] / 108$ of $\lambda^{3}+P \lambda^{2}+Q \lambda+$ $R=0$ divides the $P-Q-R$ phase-space into two regions: For $D>0(D<0), A_{i j}$ displays a focal (nodal) topology [1,2]. The velocity gradient tensor shows one real eigenvalue and two complex conjugate eigenvalues for focal topologies. By contrast, the velocity gradient tensor exhibits three real eigenvalues for nodal topologies. The surface $D=0$ gives rise to two subsets $r_{1 a}$ and $r_{1 b}$ in $P-Q-R$ phase space, which are given by [1,2] $r_{1 a}=P\left(Q-2 P^{2} / 9\right) / 3-2\left(-3 Q+P^{2}\right)^{3 / 2} / 27$ and $r_{1 b}=P\left(Q-2 P^{2} / 9\right) / 3+2\left(-3 Q+P^{2}\right)^{3 / 2} / 27$. In the region $D>0, A_{i j}$ has purely imaginary eigenvalues on the surface $r_{2}$, which are given by $R=P Q$. The surfaces $r_{1 a}, r_{1 b}$, and $r_{2}$, where $r_{2}$ is described by $P Q-R=0$, divide the $P-Q-R$ phase space into eight flow topologies, as shown in Fig. 1.

A three-dimensional DNS [19] database of freely propagating statistically planar turbulent $\mathrm{H}_{2}$-air premixed flames with $\phi=0.7$, employing a detailed chemical mechanism [20] with nine species and 19 chemical reactions, is considered here. An equivalence ratio of 0.7 is chosen because the $\mathrm{H}_{2}$-air mixture for this equivalence ratio is known to be thermodiffusively neutral [21] such that the additional effects of preferential diffusion are eliminated. The unburned gas temperature $T_{0}$ is taken to be $300 \mathrm{~K}$, which yields an unstrained laminar burning velocity $S_{L}=135.6 \mathrm{~cm} / \mathrm{s}$ under atmospheric pressure. The DNS code solves fully compressible Navier-Stokes equations where spatial discretization is carried out by an eighth-order central difference scheme for internal grid points and the order of differentiation gradually decreases to a one-sided fourth-order scheme [19]. A fourth-order Runge-Kutta scheme is used for explicit time marching [19]. The flame is initialized by a one-dimensional steady initially planar laminar flame profile [22]. A precomputed auxiliary divergence-free, homogeneous, isotropic turbulence field is generated using a pseudospectral method [23] following the Passot-Pouquet spectrum [24] and is injected through the inlet. The mean inlet velocity has been changed gradually to match turbulent flame speed as the simulation progresses. In order to assess the extent to which the flames in this study can be qualified as statistically stationary, the temporal evolution of flame area has been monitored and the flame is considered to be statistically stationary when the flame area no longer varies with time. Turbulent inflow and outflow boundaries are taken in the direction of mean flame propagation and transverse boundaries are taken to be periodic. The nonperiodic boundaries are specified using an improved Navier-Stokes characteristic boundary condition technique [25].

The inflow values of normalized root-mean-square turbulent velocity fluctuation $u^{\prime} / S_{L}$, turbulent length scale to flame thickness ratio $l_{T} / \delta_{t h}$, Damköhler number Da $=l_{T} S_{L} / u^{\prime} \delta_{t h}$, Karlovitz number $\mathrm{Ka}=\left(\rho_{0} S_{L} \delta_{t h} / \mu_{0}\right)^{0.5}\left(u^{\prime} / S_{L}\right)^{1.5}\left(l_{T} / \delta_{t h}\right)^{-0.5}$, and turbulent Reynolds number $\operatorname{Re}_{t}=\rho_{0} u^{\prime} l_{T} / \mu_{0}$ for all cases are presented in Table I, where $\mu_{0}$ is the unburned gas viscosity, $\delta_{t h}=\left(T_{a d}-T_{0}\right) / \max |\nabla T|_{L}$ is the thermal flame thickness, and the subscript $L$ is used to refer to unstrained laminar flame quantities. The turbulent length scale $l_{T}$ is the most energetic scale of the Passot-Pouquet spectrum. The cases investigated in this study are nominally representative of three regimes of combustion: case A, corrugated flamelets $(\mathrm{Ka}<1)$; case $\mathrm{B}$, thin reaction zones $(1<\mathrm{Ka}<100)$; and case $\mathrm{C}$, 
TABLE I. List of inflow turbulence parameters.

\begin{tabular}{lccccc}
\hline \hline Case & $u^{\prime} / S_{L}$ & $l_{T} / \delta_{t h}$ & $\mathrm{Re}_{t}$ & $\mathrm{Da}$ & $\mathrm{Ka}$ \\
\hline $\mathrm{A}$ & 0.7 & 14.0 & 227 & 20.0 & 0.75 \\
$\mathrm{~B}$ & 5 & 14.0 & 1623 & 2.8 & 14.4 \\
$\mathrm{C}$ & 14 & 4.0 & 1298 & 0.29 & 126 \\
\hline \hline
\end{tabular}

broken reaction zone regime $(\mathrm{Ka}>100)$ [26]. It is worth noting from Table I that Ka in cases $\mathrm{A}-\mathrm{C}$ is not modified independently of $\mathrm{Da}$ and $\mathrm{Re}_{t}$ and thus the differences in behavior among cases $\mathrm{A}-\mathrm{C}$ should not be equated solely to the influences of Karlovitz number Ka. Instead, cases A-C, for the purpose of this paper, should be considered as three typical representative scenarios of the corrugated flamelet, thin reaction zone, and broken reaction zone regimes of premixed turbulent combustion, respectively.

The domain size is $20 \times 10 \times 10 \mathrm{~mm}^{3}\left(8 \times 2 \times 2 \mathrm{~mm}^{3}\right)$ in cases A and $\mathrm{B}$ (case C) and the domain has been discretized by a uniform Cartesian grid of $512 \times 256 \times 256(1280 \times 320 \times 320)$ cells. The smaller domain for case $\mathrm{C}$ is justified by the fact that the integral scale $l_{T}$ is smaller in case $\mathrm{C}$ than in cases A and B (see Table I). The grid spacing was determined by the flame resolution, ensuring about ten grid points across $\delta_{t h}$, and in all cases the Kolmogorov length scale remains bigger than the grid spacing (i.e., $\eta \geqslant 1.5 \Delta x$, where $\eta$ and $\Delta x$ are the Kolmogorov length scale and DNS grid spacing, respectively). For this resolution about seven to nine grid points reside across the thinnest species gradient. Case $\mathrm{C}$ has the highest value of turbulent Reynolds number and thus this case requires the smallest grid spacing to resolve the Kolmogorov length and flame thickness among all the cases considered here. For the purpose of computational economy a smaller computational domain than cases A and B has been chosen here for case C. Simulations have been carried out for $1.0 t_{e}, 6.8 t_{e}$, and $6.7 t_{e}$ (i.e., $t_{e}=l_{T} / u^{\prime}$ ) for cases A-C, respectively, and this simulation time remains comparable to several previous analyses [15,16,27-29].

\section{RESULTS AND DISCUSSION}

Selected regions of instantaneous nondimensional temperature $c_{T}$, normalized first invariant $P^{*}=P \times\left(\delta_{t h} / S_{L}\right)$, second invariant $Q^{*}=Q \times\left(\delta_{t h} / S_{L}\right)^{2}$, and third invariant $R^{*}=R \times\left(\delta_{t h} / S_{L}\right)^{3}$ fields when the statistics were extracted are shown in Fig. 2. Figure 2 also shows the flame location by the contour lines of $c_{T}=0.1,0.5,0.7$ overlaid on the nondimensional temperature field. The $c_{T}$ contours clearly show the increasing level of flame wrinkling as the turbulence intensity increases from cases $\mathrm{A}$ to $\mathrm{C}$. In cases $\mathrm{A}$ and $\mathrm{B}$ the $c_{T}$ isosurfaces lie close together, whereas in case $\mathrm{C}$ they are both much farther apart and the distance between neighboring isosurfaces varies greatly. This distinction is indicative of the different combustion regimes, which is often characterized in terms of the Karlovitz number. The Karlovitz number can be scaled as $\mathrm{Ka} \sim \delta_{t h}^{2} / \eta^{2}$, where $\eta$ is the Kolmogorov length scale.

Since $\delta_{t h}$ remains smaller than $\eta$ in case $\mathrm{A}\left(\delta_{t h} \approx 0.9 \eta\right.$ and $\left.\delta_{r} \approx 0.1 \eta\right)$, the flame undergoes no significant velocity fluctuations, which are dissipated at scales of the flame thickness. Eddies with sizes above the Kolmogorov scale wrinkle the flame. Since the reaction zone remains much thinner $\left(\delta_{r} \sim 0.1 \delta_{t h}\right)$ it retains its quasilaminar structure. In case $\mathrm{B}$, on the other hand $\left(\delta_{t h} \approx 3.8 \eta\right.$ and $\delta_{r} \approx 0.4 \eta$ ), eddies with sizes smaller than 4 times the Kolmogorov scale modify the internal structure of the flame thermal thickness, while the rest of the larger eddies only wrinkle the flame. Case $\mathrm{C}$ represents the broken reaction zone regime $\left(\delta_{t h} \approx 11.2 \eta\right.$ and $\left.\delta_{r} \approx 1.1 \eta\right)$, in which eddies with sizes smaller than 11 times the Kolmogorov scale modify the internal structure of the flame thermal thickness and the eddies at the Kolmogorov scale might modify the internal structure of the reaction thickness. Eddies with sizes greater than 11 times the Kolmogorov scale wrinkle the flame strongly, resulting in a considerable distortion of flame structure. This is evident from significant 
Case A
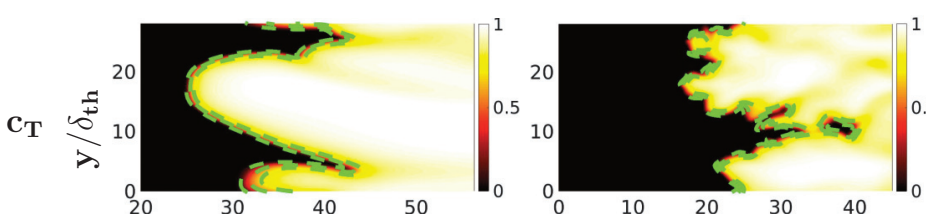

40
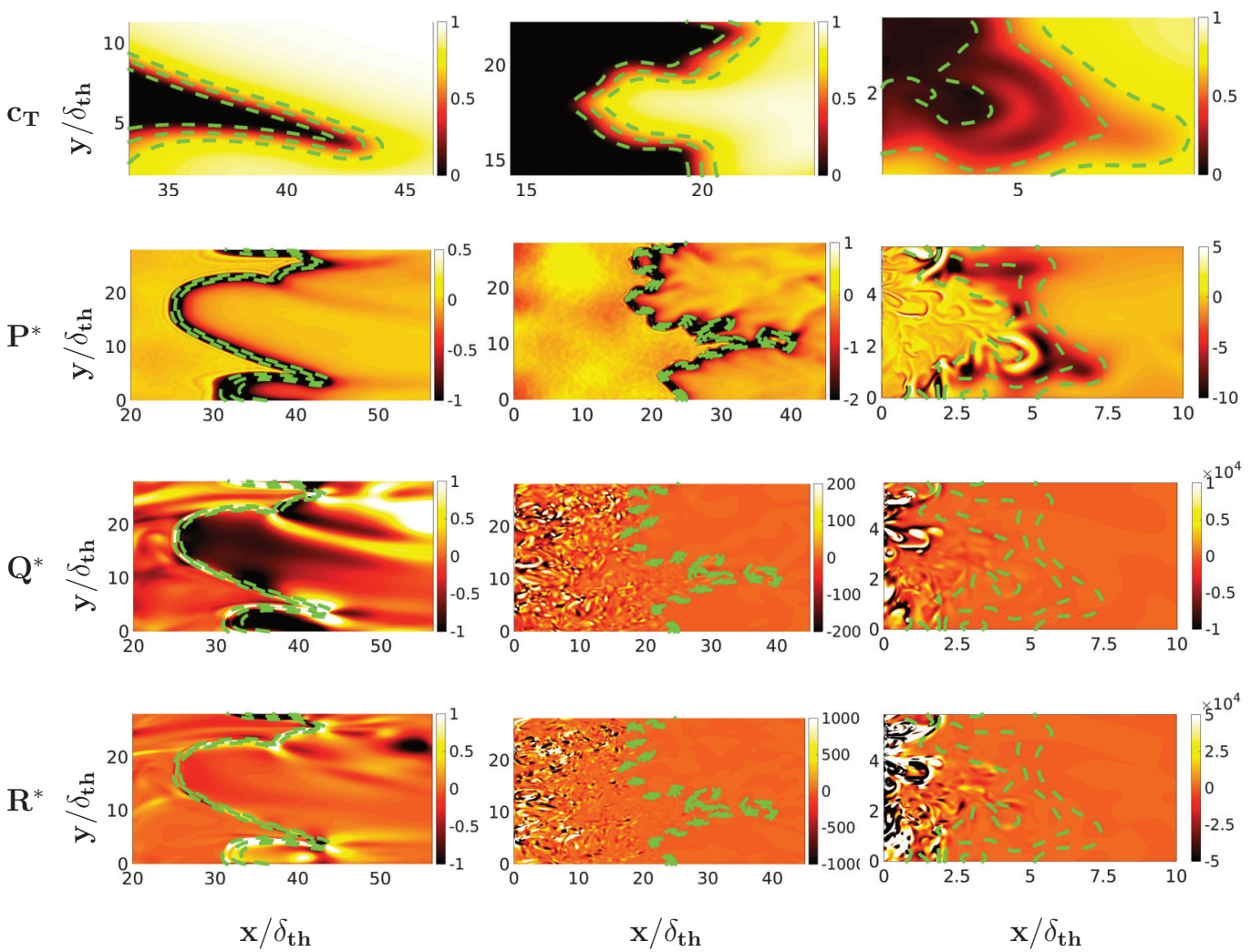

FIG. 2. Selected regions of instantaneous nondimensional temperature $c_{\mathrm{T}}$ (row 1) (green contours show $c_{T}=0.1,0.5,0.7$ isolines from left to right, shown close up in row 2), normalized first invariant $P^{*}=$ $P \times\left(\delta_{t h} / S_{L}\right)$ (row 3), second invariant $Q^{*}=Q \times\left(\delta_{t h} / S_{L}\right)^{2}$ (row 4$)$, and third invariant $R^{*}=R \times\left(\delta_{t h} / S_{L}\right)^{3}$ (row 5) fields at the $x-y$ midplane for (from left to right) cases A-C.

thickening of the flame and the large variations in the local flame thickness depending on the local turbulent flow conditions.

Note that there are no signs of localized flame extinction in case $\mathrm{C}$ in spite of large values of the Karlovitz number (i.e., $\mathrm{Ka}>100$ ). This is consistent with several previous DNS findings [30-33]. The above discussion suggests that distinctly different physical mechanisms are likely to govern the behavior of invariants in the three cases considered. It is also important to note from Table I that the values of $\mathrm{Da}, \mathrm{Ka}$, and $\mathrm{Re}_{t}$ change from one case to another here and $\mathrm{Ka}$ is not modified in isolation. Thus, the alternations of $\mathrm{Da}$ and $\mathrm{Re}_{t}$ in addition to the modification of Ka play a significant role in the differences in behavior of the invariants and their components among cases A-C.

Figure 2 shows that the qualitative nature of the distributions of $Q^{*}$ and $R^{*}$ in cases $\mathrm{B}$ and $\mathrm{C}$ is significantly different in comparison to case A: The distributions of $Q^{*}$ and $R^{*}$ in cases $\mathrm{B}$ and $\mathrm{C}$ exhibit much smaller length scales than in case A. Note that case B has much higher turbulent 
Reynolds number $\mathrm{Re}_{t}$ than in case $\mathrm{A}$ and thus case $\mathrm{B}$ shows a larger range of length scales than in case A. Cases A and B have the same values of $l_{T}$, but case B shows smaller structures of turbulence in $Q^{*}$ and $R^{*}$ distributions due to larger scale separation arising from a higher value of $\operatorname{Re}_{t}$. Case $\mathrm{C}$ has a smaller value $l_{T}$ and a greater value of $\mathrm{Re}_{t}$ than in case $\mathrm{A}$ and thus case $\mathrm{C}$ exhibits much smaller turbulence structures in $Q^{*}$ and $R^{*}$ distributions than in case A due to the combination of higher $\operatorname{Re}_{t}$ and smaller $l_{T}$.

It is also seen from Fig. 2 that large nonzero values of $P$ are concentrated within the flame because $P$ is directly related to the dilatation rate (i.e., $P=-\nabla \cdot \vec{u}$ ) and the effects of thermal expansion and dilatation rate are strong only within the flame. Furthermore, focusing of heat gives rise to high magnitudes of positive $\nabla \cdot \vec{u}$ (i.e., negative values of $P$ with high magnitude) at the locations where the flame is concave towards the reactants. In contrast, defocusing of heat leads to small positive and in some extreme cases negative values of $\nabla \cdot \vec{u}$ (i.e., small negative and positive values of $P$ ) at the locations where the flame is convex towards the reactants. ${ }^{1}$

Both the strain rate and vorticity along with the dilatation rate (i.e., $-P$ ) contribute towards the value of $Q$ since $Q_{S}=0.5\left(P^{2}-S_{i j} S_{i j}\right)$ and $Q_{W}=W_{i j} W_{i j} / 2$ according to $Q=Q_{S}+Q_{W}$ [see Eq. (1)]. The sign of $Q$ is indicative of vorticity-dominated regions outside the flame (where $P \approx 0$ for low Mach number flows like the one considered here), for which $Q>0$, and strain-dominated regions, for which $Q<0$. Figure 2 shows that both vorticity-dominated and strain-dominated regions exist in all cases outside the flame, but the degree of intermittency is much greater in cases $\mathrm{B}$ and $\mathrm{C}$ than in case A. A comparison of the magnitudes of $P$ and $Q$ from Fig. 2 reveals that the magnitude of $P^{2}$ remains smaller than the magnitude of $Q$ in most cases in the flow field and these quantities become comparable only within the flame. An increase in $u^{\prime} / S_{L}$ leads to an increase in the magnitude of $Q$ : The maximum value of $Q$ in case $\mathrm{C}$ is greater than that in case $\mathrm{B}$, which is greater than that in case A.

Distinct modes of the flame-turbulence interaction are evident by comparing cases B and C in Fig. 2 by the evolution of the $Q$ fields across the flame. The magnitude of $Q$ drops significantly across the flame in case B (see the drop of the magnitude of $Q$ across the $c_{T}=0.1$ isosurface in case B in Fig. 2). In contrast, in case $\mathrm{C}$ the magnitude of the $Q$ field remains significant throughout the flame front even beyond $c_{T}=0.7$. Finally, the high values of $Q$ are found close to the highly concave (to the reactants) regions in case A. This behavior arises due to focusing of heat in these regions, leading to an increased volumetric dilation rate $(\nabla \cdot \vec{u})$ and subsequently $Q$ due to high values of $P^{2}$ (since $P=-\nabla \cdot \vec{u}$ ) [see Eq. (1)].

The expression for $R$ contained in Eq. (1) may be rewritten as the sum of the terms that play roles in dissipation rate generation $\left(-S_{i j} S_{j k} S_{k i} / 3\right)$ and enstrophy production $\left(P Q_{W}-\omega_{i} S_{i j} \omega_{j} / 4\right)$ in the following manner:

$$
\begin{aligned}
R & =\frac{1}{3}\left(-P^{3}+3 P Q-S_{i j} S_{j k} S_{k i}\right)-\frac{1}{4} \omega_{i} S_{i j} \omega_{j} \\
& =\underbrace{\frac{1}{3}\left(-P^{3}+3 P Q_{s}-S_{i j} S_{j k} S_{k i}\right)}_{R_{s}}+P Q_{W}-\frac{1}{4} \omega_{i} S_{i j} \omega_{j} .
\end{aligned}
$$

Hence, $R^{*}$ may assume high positive or negative values where there is an imbalance of the terms contributing to dissipation rate generation and production of enstrophy. It is evident from Fig. 2 that, in case A, this imbalance is most pronounced in the vicinity of the flame front, whereas in both cases $\mathrm{B}$ and $\mathrm{C}$ it is evident throughout the entire unburned gas region. In all three cases the magnitude of $R^{*}$ is negligible in most of the burned gas region. Furthermore, in case A, the non-negligible values of $R^{*}$ retain the same sign along most of the flame front shown here, whereas, in cases B and C, both positive and negative values of $R^{*}$ coexist in the unburned gas region and within the flame front.

\footnotetext{
${ }^{1}$ It is not clearly evident from the plane shown in Fig. 2, but this occurs on other planes.
} 


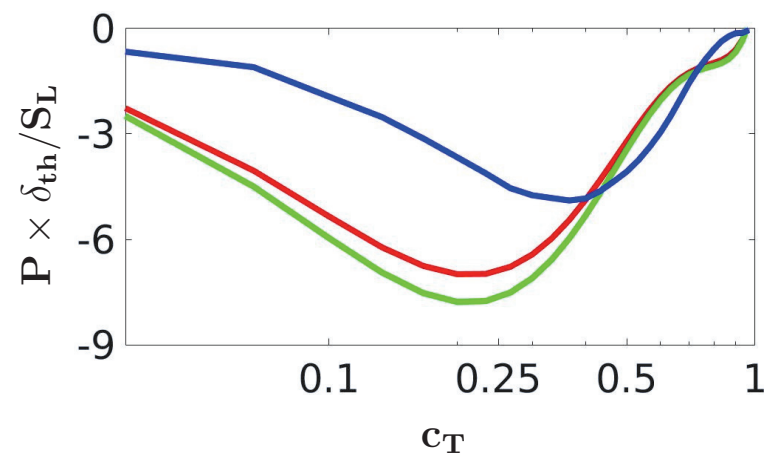

FIG. 3. Log-linear variation of $P \times\left(\delta_{t h} / S_{L}\right)$ with $c_{T}$ for cases A (red), B (green), and C (blue).

Figures 3-6 show the variations of the normalized mean values of $P, Q$, and $R$ and their constituent terms conditional on $c_{T}$. The dilatation rate $\nabla \cdot \vec{u}=-P$ remains predominantly positive in turbulent premixed flames, but it is possible to get some localized pockets of negative dilatation rate (i.e., $P>0$ ) in the regions of the flame that are convex to the reactants (see Fig. 2). However, the probability of finding a negative dilatation rate remains smaller than obtaining positive $\nabla \cdot \vec{u}$ and thus the mean value of $P=-\nabla \cdot \vec{u}$ remains negative for all cases considered here (see Fig. 3). The magnitude of $P=-\nabla \cdot \vec{u}$ depends on the strength of chemical activity within the flame. Cases A and $\mathrm{B}$ exhibit similar variations of $P$ across the flame, but the magnitudes are much less in case $\mathrm{C}$. The flame thicknesses in cases A and B are smaller or comparable to the respective $\eta$ such that the reaction zone remains quasilaminar and largely unaffected by turbulent fluctuations. Thus, although the turbulence intensity $u^{\prime} / S_{L}$ of case B is almost an order of magnitude greater than that in case $\mathrm{A}$, the thermal expansion experienced in both cases is remarkably similar. In case $\mathrm{C}$, however, the turbulent eddies enter into the inner reaction layer of the flame and disrupt the chemical processes, leading to enhanced heat loss to the preheat zone and a reduction in the reaction rate. For this reason the magnitude of $P=-\nabla \cdot \vec{u}$ observed in case $\mathrm{C}$ is much lower than that observed in cases $\mathrm{A}$ and $\mathrm{B}$. The diminishing strength of dilatation rate $\nabla \cdot \vec{u}=-P$ with increasing $\mathrm{Ka}$ is consistent with the modeling assumption by Peters [26].

Figure 4 shows the variation of mean values of $Q \times\left(\delta_{t h} / S_{L}\right)^{2}$ and its components $\left\{Q_{S}, Q_{W}\right\} \times\left(\delta_{t h} / S_{L}\right)^{2}$, conditional on $c_{T}$. Both $\nabla \cdot \vec{u}=-P$ and $\sqrt{S_{i j} S_{i j}}$ influence the component $Q_{S}=\left(P^{2}-S_{i j} S_{i j}\right) / 2$, whereas the component $Q_{W}=W_{i j} W_{i j} / 2$ depends on enstrophy $\Omega$ (i.e., $W_{i j} W_{i j} / 2=\omega_{i} \omega_{i} / 4=\Omega / 2$, where $\omega_{i}$ is the $i$ th component of vorticity). Since the turbulence intensity $u^{\prime} / S_{L}$ in case $\mathrm{A}$ is low, the main nonzero contribution arises due to $P$ as a result of thermal expansion. For case $\mathrm{A}$, the mean value of $Q_{W}$ is negligible and the mean variation of $Q$ is

Case A

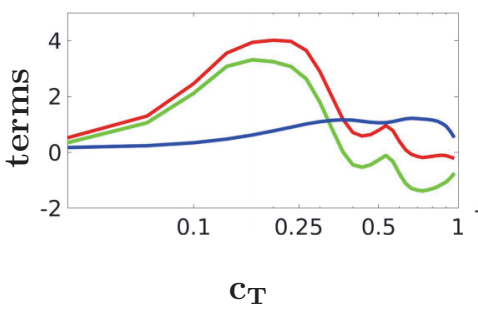

Case B

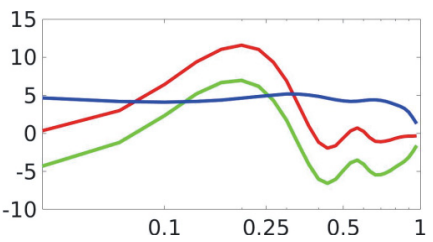

$\mathbf{C}_{\mathbf{T}}$
Case C

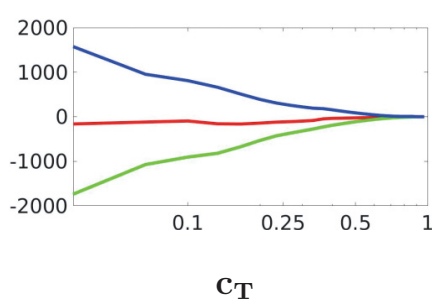

FIG. 4. Log-linear variation of $Q \times\left(\delta_{t h} / S_{L}\right)^{2}$ (red), $Q_{S} \times\left(\delta_{t h} / S_{L}\right)^{2}$ (green), and $Q_{W} \times\left(\delta_{t h} / S_{L}\right)^{2}$ (blue) with $c_{T}$ for cases A-C. 
Case A

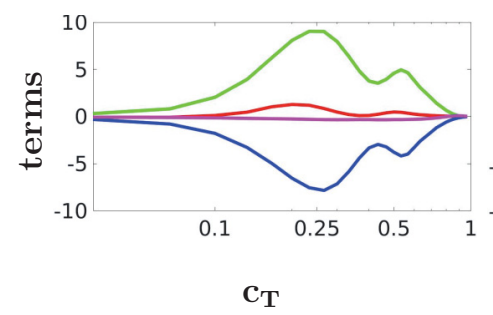

Case B

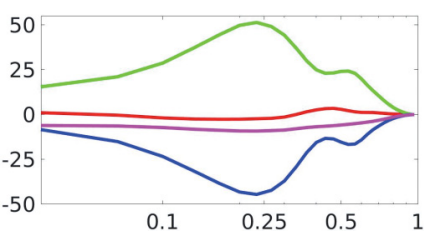

$\mathbf{c}_{\mathrm{T}}$
Case C

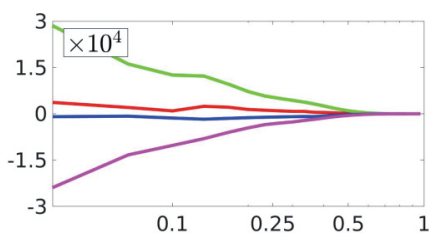

$\mathbf{c}_{\mathrm{T}}$

FIG. 5. Log-linear variation of $R \times\left(\delta_{t h} / S_{L}\right)^{3}$ (red), $R_{S} \times\left(\delta_{t h} / S_{L}\right)^{3}$ (green), $P Q_{W} \times\left(\delta_{t h} / S_{L}\right)^{3}$ (blue), and $\left(-\omega_{i} S_{i j} \omega_{j} / 4\right) \times\left(\delta_{t h} / S_{L}\right)^{3}$ (magenta) with $c_{T}$ for cases A-C. All terms in case C are to be multiplied by $10^{4}$ as indicated.

dominated by the mean value of $Q_{S}$, which assumes predominantly positive values except towards the burned gas side of the flame where the effects of $P^{2}$ are negligible due to the weak dilatation rate $\nabla \cdot \vec{u}=-P$. For case B, the mean value of $Q_{W}$ remains nonzero and positive across the flame, whereas the mean value of $Q_{S}$ remains negative for low and high values of $c_{T}$, indicating that $S_{i j} S_{i j}$ dominates over $P^{2}$. This behavior originates from weak contributions of dilatation rate $\nabla \cdot \vec{u}=-P$ on both unburned and burned gas sides of the flame (see Fig. 3) and thus the contribution of $Q_{S}=\left(P^{2}-S_{i j} S_{i j}\right) / 2$ is principally governed by $S_{i j} S_{i j}$ in these regions. However, $P^{2}$ assumes high values due to large values of dilatation rate $\nabla \cdot \vec{u}=-P$ close to the reaction zone (see Fig. 3 ) and thus $P^{2}$ dominates over $S_{i j} S_{i j}$ to give rise to positive mean value of $Q_{S}$ for intermediate values of $c_{T}$ in case B. Thus, the mean value of $Q \times\left(\delta_{t h} / S_{L}\right)^{2}$ remains small at high and low values of $c_{T}$ and attains its maximum values at $c_{T} \approx 0.2$, where the mean values of $Q_{S}$ and $Q_{W}$ are both positive. In contrast to cases $\mathrm{A}$ and $\mathrm{B}$, the magnitude of $Q$ and its components at $c_{T} \approx 0.0$ are far greater in case C. The mean contribution of $Q_{S}=\left(P^{2}-S_{i j} S_{i j}\right) / 2$ remains negative throughout the flame in case $\mathrm{C}$ because in this case the effects of the dilatation rate $\nabla \cdot \vec{u}=-P$ are too weak to supersede the influences of $S_{i j} S_{i j}$ and thus in this case the behavior of $Q_{S}$ is principally governed by $\left(-S_{i j} S_{i j}\right) / 2$.

The quantity $Q_{S}$ can be expressed as $Q_{S}=Q_{S 1}+Q_{S 2}=P^{2} / 3-E / 4 v$, where $E=$ $\left(\tau_{i j} \partial u_{i} / \partial x_{j}\right) / \rho$ is the dissipation rate of instantaneous kinetic energy (i.e., $\left.u_{i} u_{i} / 2\right)$ and $v$ is the kinematic viscosity. Hence, $Q_{S}>0\left(Q_{S}<0\right)$ corresponds to dilation- (dissipation-) dominated regions. Using $Q_{S 1}=P^{2} / 3 \sim\left\{\tau S_{L} / \delta_{t h}\right\}^{2}[17,34]$ and $\left|Q_{S 2}\right|=|-E / 4 \nu| \sim 1 / \tau_{\eta}^{2}$ (where $\tau_{\eta}$ is the Kolmogorov time scale) leads to $Q_{S 1} /\left|Q_{S 2}\right| \sim \tau^{2} \mathrm{Ka}^{-2}$, where $\tau=\left(T_{a d}-T_{0}\right) / T_{0}$ is the heat release parameter. This suggests that the relative strength of $Q_{S 1}$ with respect to $Q_{S 2}$ weakens (strengthens) with increasing $\mathrm{Ka}$ (heat release). Thus, in case $\mathrm{C}$ (where $\mathrm{Ka} \gg 1$ ), the mean behavior of $Q_{S}$ is governed by $Q_{S 2}=-E / 4 v$, whereas in case $\mathrm{A}$ (where $\mathrm{Ka}<1$ ) the mean behavior of $Q_{S}$ is governed by the positive mean value of $Q_{S 1}=P^{2} / 3$ for the major part of the flame brush. In case B (where

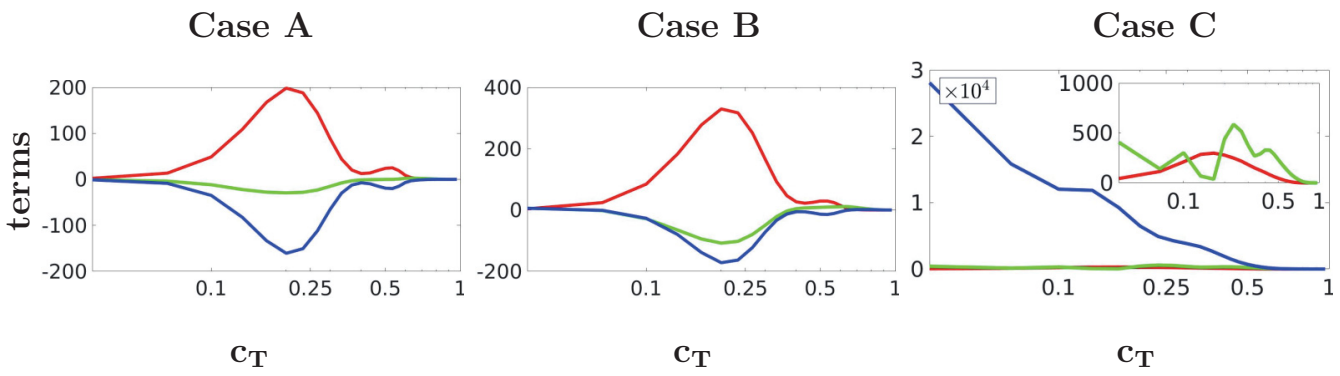

FIG. 6. Log-linear variation of $\left\{-P^{3} / 3\right\} \times\left(\delta_{t h} / S_{L}\right)^{3} \quad\left(\right.$ red), $\quad\left\{P Q_{S}\right\} \times\left(\delta_{t h} / S_{L}\right)^{3} \quad$ (green), and $\left\{-S_{i j} S_{j k} S_{k i} / 3\right\} \times\left(\delta_{t h} / S_{L}\right)^{3}$ (blue) with $c_{T}$ for cases A-C. The inset in case C shows variation of terms of smaller magnitude. All terms in case $\mathrm{C}$ are to be multiplied by $10^{4}$ as indicated. 
Ka $>1$ ), $Q_{S 1}$ dominates over $Q_{S 2}$ to result in a mean positive value of $Q_{S}$ only in the region of the flame where the effects of heat release are strong.

In summary, in case $\mathrm{C}$ the mean values of $Q_{S}$ and $Q_{W}$ largely balance each other, although the mean value of $Q$ remains negative across the entire flame front, which is opposite to the behavior of cases $\mathrm{A}$ and $\mathrm{B}$.

The third invariant $R$ may be written as $R=R_{S}+P Q_{W}-\omega_{i} S_{i j} \omega_{j} / 4$, where $R_{S}=\left(-P^{3}+\right.$ $\left.3 P Q_{S}-S_{i j} S_{j k} S_{k i}\right) / 3$ contains a contribution to the dissipation rate generation (i.e., $S_{i j} S_{j k} S_{k i}$ ), while $\left(P Q_{W}-\omega_{i} S_{i j} \omega_{j} / 4\right)$ contributes to the enstrophy production rate $[6,16]$. Thus, $R>0$ indicates that the enstrophy production rate dominates over the dissipation rate generation and vice versa $[6,16]$. The contributions of $P Q_{W}-\omega_{i} S_{i j} \omega_{j} / 4$ and $R_{S}$ appear to balance across the flame front in cases $\mathrm{A}$ and $\mathrm{B}$ (see Fig. 5). In both cases the mean value of $R_{S}$ attains its maximum value, whereas the mean value of $P Q_{W}$ attains its minimum value, at $c_{T} \approx 0.25$. In case $\mathrm{C}$, the mean value of $R_{S}$ is largely balanced by the mean contribution of $-\omega_{i} S_{i j} \omega_{j} / 4$, which are both an order of magnitude greater than the mean values of $P Q_{W}$ and $R$. The mean value of $R$ attains its maximum value at $c_{T} \approx 0.0$, but also a local maximum is obtained at $c_{T} \approx 0.20$, and $R$ remains positive across the flame, indicating that the enstrophy production rate dominates over the dissipation rate generation, unlike cases $\mathrm{A}$ and $\mathrm{B}$ where the mean $R \approx 0$ across the flame.

Finally, Fig. 6 shows the individual terms contributing to $R_{S}:-P^{3} / 3, P Q_{S}$, and $-S_{i j} S_{j k} S_{k i} / 3$. For cases $\mathrm{A}$ and $\mathrm{B}$, all terms are approximately zero at $c_{T} \approx 0.0$ and $c_{T} \approx 1.0$. The mean value of $-P^{3} / 3$ remains positive and is balanced by the mean contributions of $P Q_{S}$ and $-1 / 3 S_{i j} S_{j k} S_{k i}$, which are both negative in the region $0.15<c_{T}<0.35$ for cases $\mathrm{A}$ and $\mathrm{B}$. In contrast, in case $\mathrm{C}$, the mean contributions of $-P^{3} / 3, P Q_{S}$, and $-S_{i j} S_{j k} S_{k i} / 3$ are positive, but they are dominated by the mean value of $-S_{i j} S_{j k} S_{k i} / 3$, which is related to the dissipation rate generation. One can express $(\nabla \cdot \vec{u}) E$ as $(\nabla \cdot \vec{u}) E=4 v\left(P Q_{S}-P^{3} / 3\right)$. It can be seen from the mean values of $P Q_{S}$ and $-P^{3} / 3$ in Fig. 6 that the mean value of $P Q_{S}-P^{3} / 3=(\nabla \cdot \vec{u}) E / 4 v$ remains positive throughout the flame. As the mean value of $\nabla \cdot \vec{u}$ remains positive and the correlation between $\nabla \cdot \vec{u}$ and $E$ is not particularly strong, the positive mean value of $P Q_{S}-P^{3} / 3$ in Fig. 6 indicates a positive mean value of $E=2 v\left(S_{i j} S_{i j}-P^{2} / 3\right)$.

Figure 7 shows the joint probability density function (PDF) contours of the normalized second and third invariants $F\left(Q^{*}, R^{*}\right)$ for cases A-C on $c_{T}=0.1,0.5,0.7$, where $Q^{*}=Q \times\left(\delta_{t h} / S_{L}\right)^{2}$ and $R^{*}=R \times\left(\delta_{t h} / S_{L}\right)^{3}$. The behavior on isosurfaces of higher $c_{T}$ values is not shown here, since $Q^{*} \approx 0.0$ for higher values of $c_{T}$ (see Fig. 4). The joint PDF exhibits a negative correlation between $Q^{*}$ and $R^{*}$ [2,5] for all $c_{T}$ isosurfaces. As $c_{T}$ is increased, the most probable value of the distribution moves towards the origin (i.e., $Q^{*}=0$ and $R^{*}=0$ ).

It is useful to examine the variation of the individual local topologies $S 1-S 8$ across the flame and to see how their variation changes from one case to another. Figures 8 (a)-8(c) show the variation of the volume fraction (VF) of each topology as a function of $c_{T}$ following the approach adopted by Cifuentes et al. $[15,16]$. Figure 8 reveals a noticeable difference in the distribution of the flow topology between cases A-C. For case A, both focal and nodal topologies show clear variation with $c_{T}$, with $S 1, S 3$, and $S 4$ increasing and $S 2, S 7$, and $S 8$ decreasing as moving from the unburned to the burned gas region. Such trends diminish from case A to case $\mathrm{C}$ such that each topology is more uniformly distributed across $c_{T}$ in case $\mathrm{C}$. In particular, for case $\mathrm{C}$, the $S 8$ nodal topology disappears entirely. The $S 8$ topology is associated with high positive values of dilatation rate $(\nabla \cdot \vec{u}=-P \gg 0)$ and thus its probability decreases for case $\mathrm{C}$ due to weakening of dilatation rate.

Figure 8(d) compares the distributions of volume fraction of total combined focal (i.e., $S 1, S 4$, $S 5$, and $S 7$ ) and nodal (i.e., $S 2, S 3, S 6$, and $S 8$ ) topologies between cases A-C. For case A nodal topologies are dominant in the unburned gas region and focal topologies in the burned gas region. This contrasts with the previous simple chemistry analyses $[15,16]$, which showed that the VF of focal topologies decreases from the unburned gas to the burned gas side. The heat release parameter $\tau=\left(T_{a d}-T_{0}\right) / T_{0}$ for cases A-C is greater than that used in Refs. [15,16] (6 as opposed to 4) and thus the flame-induced turbulence is stronger in these cases than in the cases analyzed in 

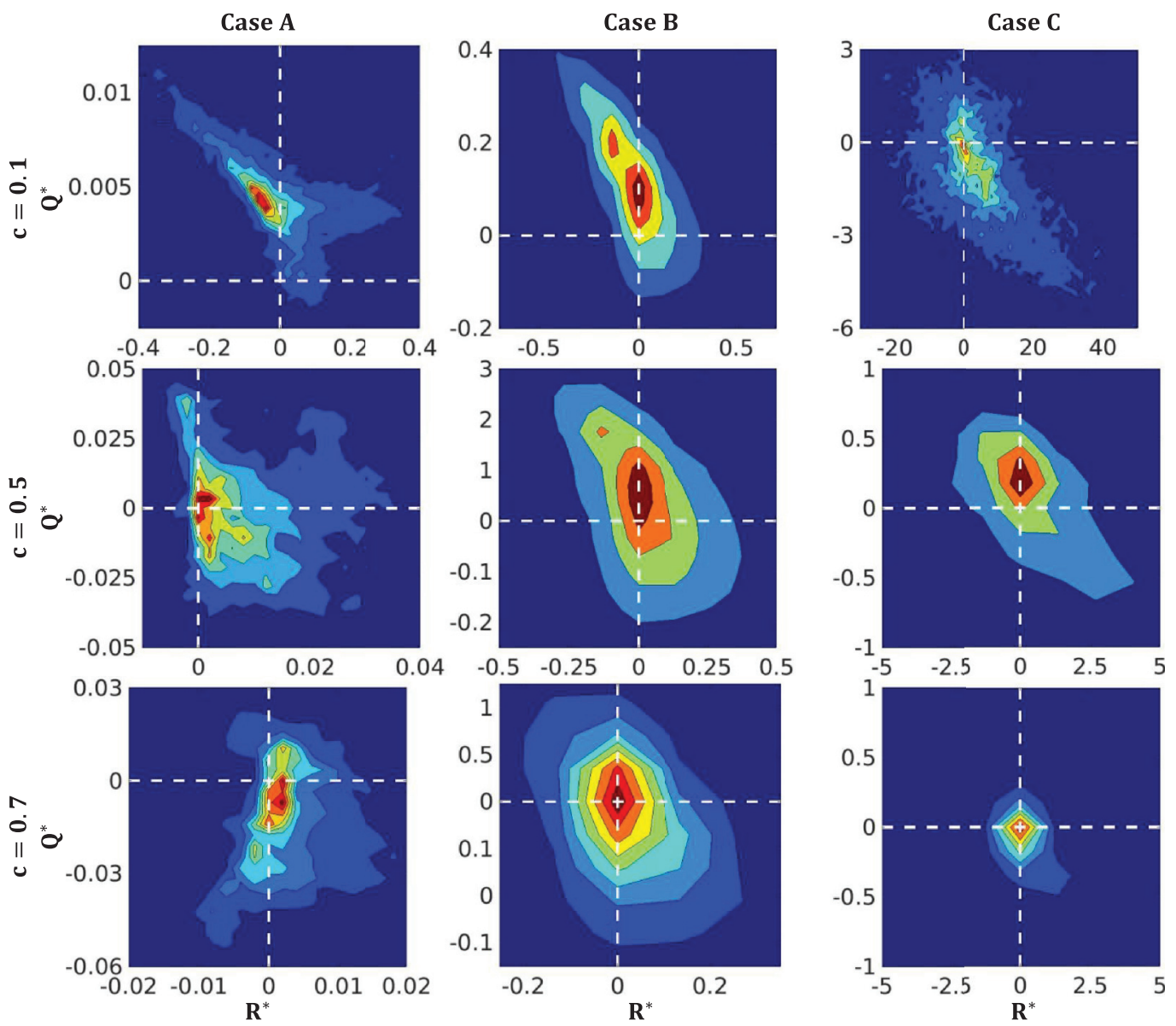

FIG. 7. Joint PDFs of $Q^{*}=Q \times\left(\delta_{t h} / S_{L}\right)^{2}$ and $R^{*}=R \times\left(\delta_{t h} / S_{L}\right)^{3}, F\left(Q^{*}, R^{*}\right)$, on $c_{T}=0.1,0.5,0.7$ isosurfaces for cases A-C. The value of $F\left(Q^{*}, R^{*}\right)$ increases from blue to red color.

Refs. [15,16], where the VF of vortical (focal) topologies decayed across the flame. ${ }^{2}$ The strength of vortical structures within the flame front increases within the flame due to flame-induced turbulence in case A, which is reflected in the increase in the VF of focal (i.e., vortical) topologies within the flame. In case $\mathrm{C}$, the flame does not significantly influence the background turbulent flow field and the focal topologies remain dominant across the entire flamefront.

The statistics of flame curvature plays a key role in order to understand the interrelation between the distributions of the flow and flame topologies. The topology of a $c_{T}$ isosurface can be described in terms of its mean and Gauss curvatures $\kappa_{m}$ and $\kappa_{g}$, respectively, following Dopazo et al. [7], where $\kappa_{m}=\left(\kappa_{1}+\kappa_{2}\right) / 2=1 / 2 \nabla \cdot\left(-\nabla c_{T} /\left|\nabla c_{T}\right|\right)$ and $\kappa_{g}=\kappa_{1} \kappa_{2}$, in which $\kappa_{1}$ and $\kappa_{2}$ are the principal curvatures $[7,16]$. In the $\kappa_{m}-\kappa_{g}$ plane, the region $\kappa_{g}>\kappa_{m}^{2}$ indicates complex curvatures and thus is nonphysical. Moreover, positive (i.e., $\kappa_{m}>0$ ) curvature is associated with the wrinkles that are convex to the reactants, whereas negative (i.e., $\kappa_{m}<0$ ) curvature represents wrinkles that are

\footnotetext{
${ }^{2}$ The generation of enstrophy due to baroclinic torque is indeed found to be comparable to the magnitude of viscous dissipation of enstrophy in case $\mathrm{A}$, which leads to considerable enstrophy generation within the flame for this case. By contrast, the viscous dissipation of enstrophy dominates over baroclinic torque contribution in cases $\mathrm{B}$ and $\mathrm{C}$, where the vortex-stretching and viscous dissipation remain the leading-order contributors.
} 
(a)

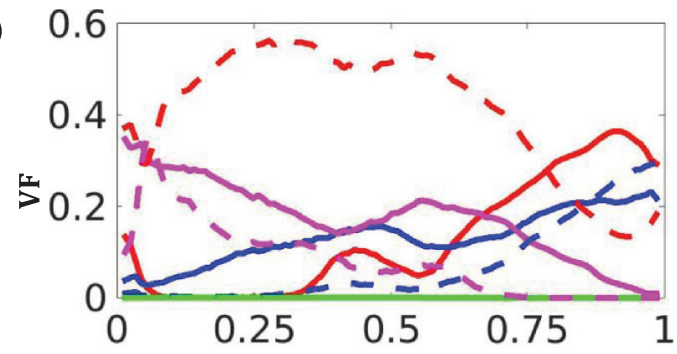

(b)

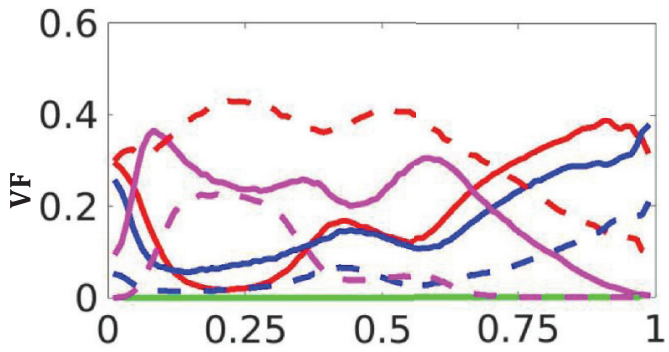

(c)

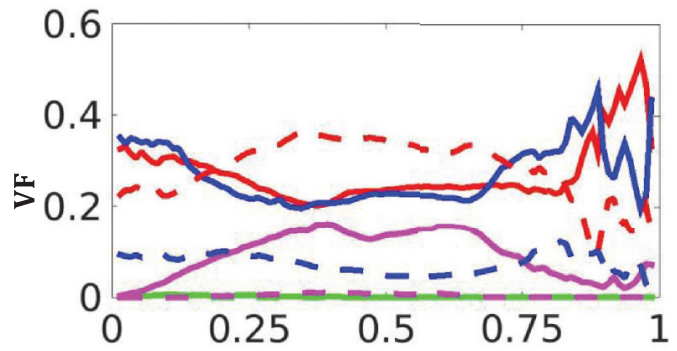

(d)

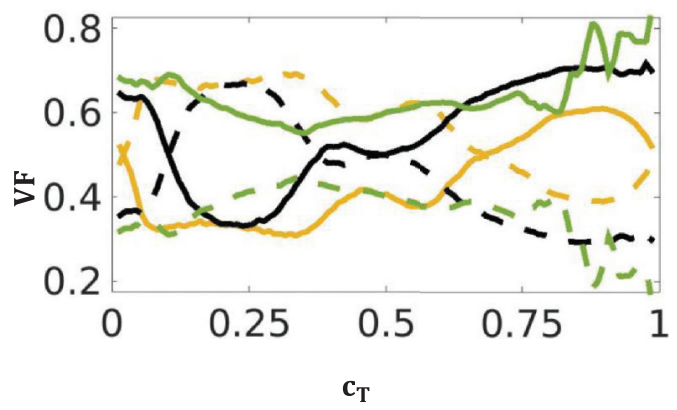

FIG. 8. Variation of volume fractions of topologies $S 1-S 8$ with $c_{T}$ for (a)-(c) cases A-C: focal topologies $S 1$ (red solid line), $S 4$ (blue solid line), $S 5$ (green solid line), and $S 7$ (magenta solid line) and nodal topologies $S 2$ (red dashed line), $S 3$ (blue dashed line), $S 6$ (green dashed line), and $S 8$ (magenta dashed line). (d) Variation of VFs of total focal (solid lines) and nodal (dashed lines) topologies with $c_{T}$ for cases A (tan), B (black), and $\mathrm{C}$ (olive).

concave to the reactants (see Fig. 2). The realizable part of $\kappa_{m}>0\left(\kappa_{m}<0\right)$ and $\kappa_{g}>0$ represents cup convex (cup concave) flame topology. By contrast, $\kappa_{m}>0\left(\kappa_{m}<0\right)$ and $\kappa_{g}<0$ represents saddle convex (saddle concave) flame topology. The combination of $\kappa_{m}>0\left(\kappa_{m}<0\right)$ and $\kappa_{g}=0$ represents tile convex (concave) flame topology. Figure 9 shows a scatter plot of the mean versus Gaussian curvature for cases A-C conditional on one representative focal (S7) and one nodal (S3) topology. The plots in Fig. 9 are colored to highlight the highest concentrations of data points. It is apparent from Fig. 9 that the distribution of topologies $S 3$ and $S 7$ favors $\kappa_{m}, \kappa_{g}>0\left(\kappa_{m}<0\right.$ and $\kappa_{g}>0$ ) for case A (for case C), whereas case B shows a more symmetric distribution. A similar 

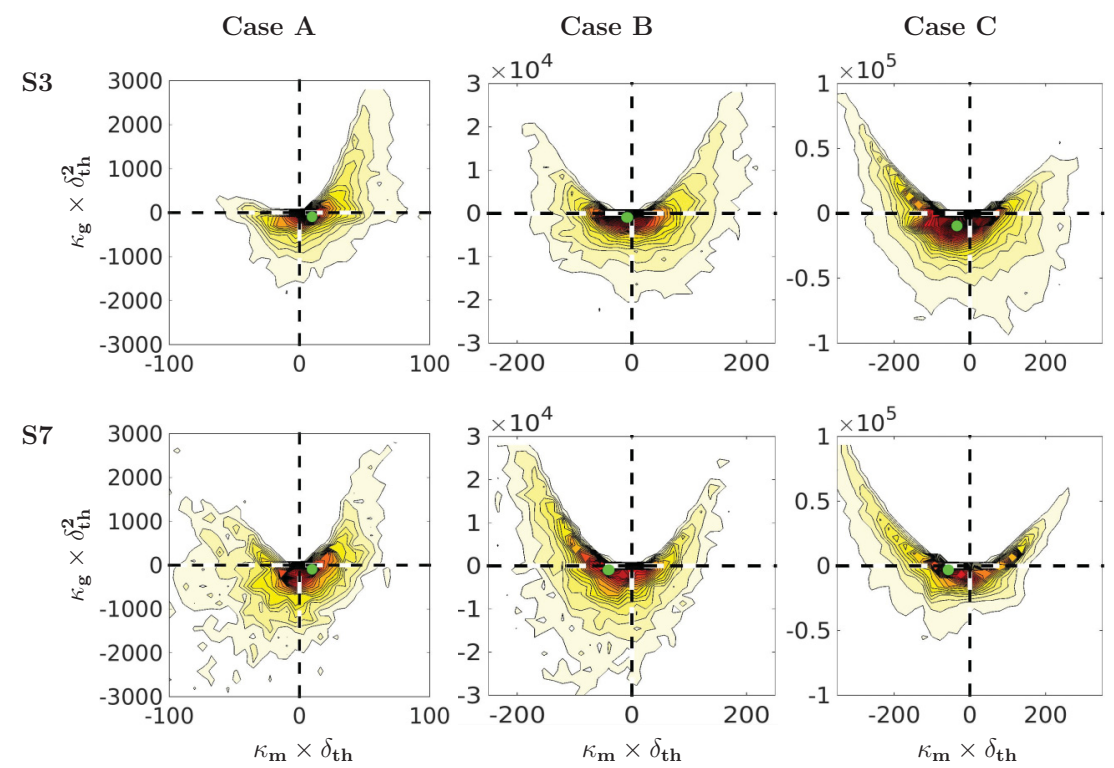

FIG. 9. Scatter of normalized mean and Gaussian curvatures colored by count for (from left to right) cases A-C. Topologies $S 3$ and $S 7$ are shown exemplarily. Magnitude increases with color from white to red. Green dots indicate the location of the maximum values.

trend is observed for topologies $S 1, S 2$, and $S 8$. Topology $S 4$ is more symmetric and for topologies $S 5$ and $S 6$ there were insufficient data. These results do not reveal any consistent trend between flow and flame topologies based on these results, and a more detailed analysis is needed in this respect.

Figure 1 shows that the topologies are associated with different types of generic flow structures. Thus, the contributions of these topologies to turbulent processes such as micromixing characterized by the scalar dissipation rate (SDR) $N_{c}=D \nabla c_{T} \cdot \nabla c_{T}$ (where $D$ is the thermal diffusivity) and enstrophy $\Omega=\vec{\omega} \cdot \vec{\omega} / 2$ transport in turbulent premixed flames are of fundamental importance. The transport equations of $N_{c}$ and $\Omega$ are given by [17,18,34,35]

$$
\begin{aligned}
\rho \frac{D N_{c}}{D t}= & \frac{\partial}{\partial x_{j}}\left(\rho D \frac{\partial N_{c}}{\partial x_{j}}\right)-2 D \frac{D c_{T}}{D t} \frac{\partial c_{T}}{\partial x_{k}} \frac{\partial \rho}{\partial x_{k}}-2 \rho D \underbrace{\frac{\partial c_{T}}{\partial x_{i}} \frac{\partial u_{i}}{\partial x_{j}} \frac{\partial c_{T}}{\partial x_{j}}}_{\Lambda} \\
& +2 D \frac{\partial \dot{\omega}_{T}}{\partial x_{k}} \frac{\partial c_{T}}{\partial x_{k}}-2 \rho D^{2} \frac{\partial^{2} c_{T}}{\partial x_{k} \partial x_{i}} \frac{\partial^{2} c_{T}}{\partial x_{k} \partial x_{i}}+f(D), \\
\frac{D \Omega}{D t}= & \underbrace{\omega_{i} \omega_{k} \frac{\partial u_{i}}{\partial x_{k}}}_{V}-\epsilon_{i j k} \omega_{i} \frac{1}{\rho^{2}} \frac{\partial \rho}{\partial x_{j}} \frac{\partial \tau_{k l}}{\partial x_{l}}+\frac{\epsilon_{i j k} \omega_{i}}{\rho} \frac{\partial^{2} \tau_{k l}}{\partial x_{j} \partial x_{l}}-2 \frac{\partial u_{k}}{\partial x_{k}} \Omega+\epsilon_{i j k} \frac{\omega_{i}}{\rho^{2}} \frac{\partial \rho}{\partial x_{j}} \frac{\partial p}{\partial x_{k}}
\end{aligned}
$$

where $\rho, p, \dot{\omega}_{T}$, and $\tau_{i j}$ are the density, pressure, chemical source term, and viscous stress tensor, respectively, and $f(D)$ accounts for the contribution due to diffusivity gradients. The terms $-2 \rho D \Lambda$ and $V$ are referred to as the scalar-turbulence interaction and vortex-stretching terms, respectively $[17,18,34,35]$. The term $\Lambda$ in Eq. (3a) can be written as $\Lambda=\left(\partial c_{T} / \partial x_{i}\right)\left(\partial u_{i} / \partial x_{j}\right)\left(\partial c_{T} / \partial x_{j}\right)=$ $\left(e_{\alpha} \cos ^{2} \alpha+e_{\beta} \cos ^{2} \beta+e_{\gamma} \cos ^{2} \gamma\right) \nabla c_{T} \cdot \nabla c_{T}=a_{n} \nabla c_{T} \cdot \nabla c_{T}$, where $a_{n}=N_{i} N_{j} \partial u_{i} / \partial x_{j}$ is the normal strain rate with $N_{i}=-\left(\partial c_{T} / \partial x_{i}\right) /\left|\nabla c_{T}\right|$ being the $i$ th component of the flame normal vector [17]. This suggests that $\Lambda$ takes a positive (negative) value for positive (negative) values of $a_{n}$ [17]. The expression $\Lambda=\left(e_{\alpha} \cos ^{2} \alpha+e_{\beta} \cos ^{2} \beta+e_{\gamma} \cos ^{2} \gamma\right) \nabla c_{T} \cdot \nabla c_{T}=a_{n} \nabla c_{T} \cdot \nabla c_{T}$ indicates that the behaviors of $\Lambda$ and $a_{n}$ are governed by the alignment of $\nabla c_{T}$ with local principal strain rates. 
Case A
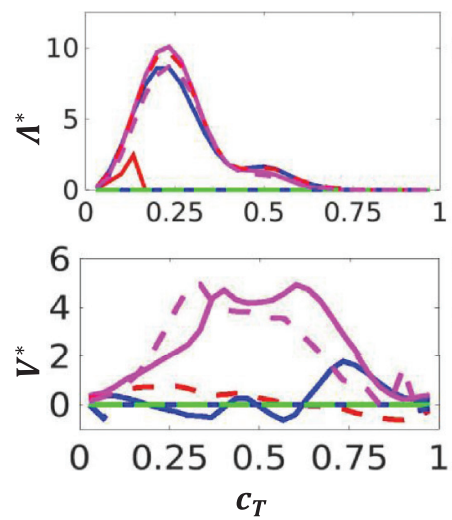

Case B
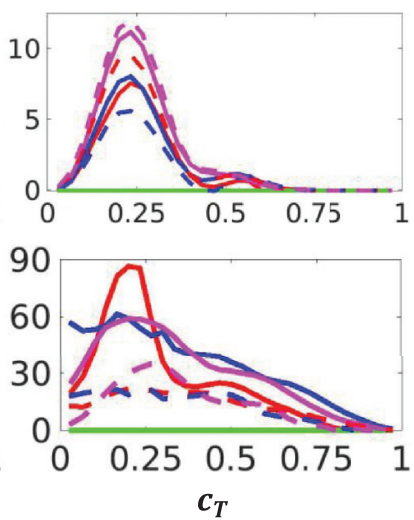

Case C
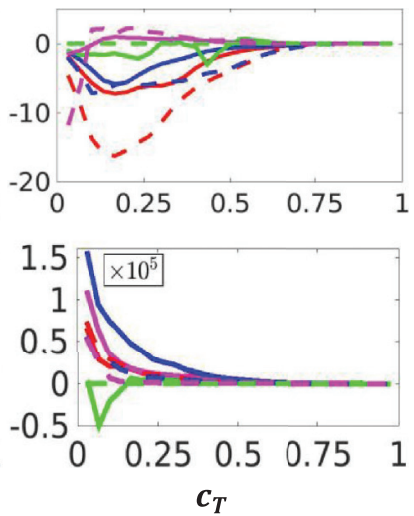

FIG. 10. Variation of the mean values of $\Lambda^{*}=\Lambda \times\left(\delta_{t h}^{3} / S_{L}\right)$ (top row) and $V^{*}=V \times\left(\delta_{t h} / S_{L}\right)^{3}$ (bottom row) conditional on $c_{T}$ for cases A (left column), B (middle column), and C (right column): focal topologies $S 1$ (red solid line), $S 4$ (blue solid line), $S 5$ (green solid line), and $S 7$ (magenta solid line) and nodal topologies $S 2$ (red dashed line), $S 3$ (blue dashed line), $S 6$ (green dashed line), and $S 8$ (magenta dashed line). Results are omitted where the volume fraction is less than 0.01 . The values of $V^{*}$ in case $\mathrm{C}$ are to be multiplied by $10^{5}$ as indicated.

As the flow topologies are associated with particular combinations of strain rate and vorticity distributions, they are likely to influence the statistical behaviors of terms $\Lambda$ and $V$. These dependences of $\Lambda=\left(\partial c_{T} / \partial x_{i}\right)\left(\partial u_{i} / \partial x_{j}\right)\left(\partial c_{T} / \partial x_{j}\right)=\left(e_{\alpha} \cos ^{2} \alpha+e_{\beta} \cos ^{2} \beta+e_{\gamma} \cos ^{2} \gamma\right) \nabla c_{T} \cdot \nabla c_{T}$ and $V=2\left(e_{\alpha} \cos ^{2} \alpha^{\prime}+e_{\beta} \cos ^{2} \beta^{\prime}+e_{\gamma} \cos ^{2} \gamma^{\prime}\right) \Omega$ arise due to the alignment of $\nabla c_{T}$ and $\vec{\omega}$ with the most extensive (i.e., most positive), intermediate, and most compressive (i.e., most negative) strain rates (i.e., $e_{\alpha}, e_{\beta}$, and $\left.e_{\gamma}\right)$, where $\alpha, \beta$, and $\gamma\left(\alpha^{\prime}, \beta^{\prime}\right.$, and $\left.\gamma^{\prime}\right)$ are the angles between $\nabla c_{T}(\vec{\omega})$ and the eigenvectors associated with $e_{\alpha}, e_{\beta}$, and $e_{\gamma}$, respectively.

The first row of Fig. 10 shows the contribution of different topologies to the mean values of $\Lambda$ conditional on $c_{T} \cdot{ }^{3}$ Cases $\mathrm{A}$ and B show positive mean contributions of $\Lambda$ for all topologies except for $S 5$ and $S 6$ in case A and $S 3, S 5$, and $S 6$ in case B. The mean value of $\Lambda$ peaks at $c_{T} \approx 0.25$. In case A, $S 2$ and $S 7$ remain major contributors of $\Lambda$, closely followed by $S 4$ and $S 8$, whereas $S 8$ is the primary contributor in case B, followed, in order, by $S 7, S 2, S 4, S 1$, and $S 3$. The absence of contributions of $S 1$ and $\mathrm{S} 3$ in case A is due to the scarcity of the corresponding samples at low values of $c_{T}$ [see Fig. 8(a)]. Although case $\mathrm{C}$ also displays peak mean values of $\Lambda$ for low $c_{T}$, the behavior of the topologies is vastly different: Mean contributions for $S 1-S 7$ are mostly negative, with the exception of $S 8$. The peak magnitude of the negative mean value of $\Lambda$ is obtained for $S 2$ at $c_{T} \approx 0.15$. A non-negligible contribution is obtained from $S 5$, although the sample size remains small [see Fig. 8(c)].

A preferential alignment between $\nabla c_{T}$ and $e_{\alpha}\left(e_{\gamma}\right)$, characterized by high probability of $\cos ^{2} \alpha \approx 1.0\left(\cos ^{2} \gamma \approx 1.0\right)$, leads to a positive (negative) $\Lambda[17,34,36-39]$. It has been shown elsewhere [17,34,36-39] that $\nabla c_{T}$ preferentially aligns with $e_{\alpha}$ when the strain rate induced by

\footnotetext{
${ }^{3}$ The components of $\Lambda$ and $V$ conditional on topology have not been shown because they deterministically show similar behavior. For example, the mean values of $\left(e_{\alpha} \cos ^{2} \alpha\right) \nabla c_{T} \cdot \nabla c_{T}$ and $2\left(e_{\alpha} \cos ^{2} \alpha^{\prime}\right) \Omega$ conditional on each topology will exhibit positive values due to positive value of $e_{\alpha}$. By the same token, the mean values of $\left(e_{\gamma} \cos ^{2} \gamma\right) \nabla c_{T} \cdot \nabla c_{T}$ and $2\left(e_{\gamma} \cos ^{2} \gamma^{\prime}\right) \Omega$ conditional on topology will deterministically exhibit negative values for all cases. The magnitudes of these conditional mean values are expected to be different from one case to another because the strain rate magnitude will depend on $u^{\prime} / S_{L}$ and $l_{T} / \delta_{t h}$.
} 
flame normal acceleration overcomes turbulence straining. In contrast, $\nabla c_{T}$ aligns with $e_{\gamma}$ under the dominance of turbulent straining [17,34,36-39]. As the effects of heat release are stronger in the corrugated flamelets and thin reaction zone regime flames (e.g., cases $\mathrm{A}$ and $\mathrm{B}$ ) than in the broken reaction zone regime flames (e.g., case $\mathrm{C}$ ), $\nabla c_{T}$ shows strong alignment with $e_{\alpha}\left(e_{\gamma}\right)$ in cases $\mathrm{A}$ and $\mathrm{B}$ (case $\mathrm{C}$ ), thus leading to a positive (negative) value of $\Lambda$. Although case $\mathrm{C}$ exhibits predominantly negative $\Lambda$, the $S 8$ topology is associated with local high positive $\nabla \cdot \vec{u}=-P$ (see Fig. 1) and thus $\nabla c_{T}$ aligns locally with $e_{\alpha}$ to result in a positive $\Lambda$ in this region.

A careful comparison reveals that the mean values of $\Lambda$ conditional on $c_{T}$ for $S 1-S 4$ topologies exhibit positive values due to predominant alignment of $\nabla c_{T}$ with $e_{\alpha}$ under the action of strong heat release in cases $\mathrm{A}$ and $\mathrm{B}$. By contrast, predominant alignment of $\nabla c_{T}$ with $e_{\gamma}$ due to weak influences of heat release in case $\mathrm{C}$ yields negative mean values of $\Lambda$ conditional on $c_{T}$ for $S 1-S 4$ topologies. As the flow topologies $S 5$ and $S 6$ are rare occurrences in premixed flames, a consistent trend is not expected for these topologies. The topologies $S 7$ and $S 8$ are associated with positive $\nabla \cdot \vec{u}=-P$ (see Fig. 1) and thus the effects of heat release are strongly felt in these topologies and as a result $\nabla c_{T}$ aligns locally with $e_{\alpha}$ to yield positive mean values of $\Lambda$ conditional on $c_{T}$ for these topologies in all three cases. However, the effects of heat release are weak towards the unburned gas side of the flame and thus the mean value of $\Lambda$ conditional on $c_{T}$ exhibits negative values even for the $S 8$ topology in case $\mathrm{C}$.

The second row of Fig. 10 shows the contribution of different topologies to the mean values of $V=2\left(e_{\alpha} \cos ^{2} \alpha^{\prime}+e_{\beta} \cos ^{2} \beta^{\prime}+e_{\gamma} \cos ^{2} \gamma^{\prime}\right) \Omega$ conditional on $c_{T}$, which reveals that the mean value of $V$ conditional on $c_{T}$ for all topologies remain positive for all three cases. The predominant alignment of $\vec{\omega}$ with the intermediate and most extensive principal strain rates (i.e., $e_{\beta}$ and $e_{\alpha}$ ) in these cases, in accordance with previous findings [18,32,35], gives rise to a positive mean value of $V$ for all cases considered here, but both the mean value and the qualitative behavior vary greatly between cases. In case A, $S 7$ and $S 8$ remain dominant contributors to $V$ between $0.25<c_{T}<0.75$, but no clear peak in their mean contribution is evident. In case $\mathrm{B}$, however, the contribution of $S 1$ is dominant and its mean value shows a clear peak at $c_{T} \approx 0.25$. Finally, in case $\mathrm{C}$, all nonzero topologies exhibit peak values at $c_{T} \approx 0.0$, with $S 4$ and $S 7$ attaining the highest mean values of $V$. Furthermore, in cases $\mathrm{B}$ and $\mathrm{C}$, with the exception of $S 5$ in case $\mathrm{C}$, the contributions of all focal topologies of significant presence (i.e., $S 1, S 4$, and $S 7$ ) attain higher values across the entire flame than for all significant nodal topologies (i.e., $S 2, S 3$, and $S 8$ ).

The effects of flame-induced turbulence are the strongest for topologies that are associated with high positive $\nabla \cdot \vec{u}=-P$ in case A (e.g., $S 7$ and $S 8$ ). This trend weakens with increasing Ka. Thus, the focal topologies associated with positive $Q[S 1, S 4$, and $S 7$ (see Fig. 1)] contribute more to $V$ than the nodal topologies $S 2, S 3$, and $S 8$. The topologies $S 4$ and $S 7$ are associated with the vortex stretching (see Fig. 1) and thus they exhibit a high positive mean contribution of $V$ in case C. In the absence of significant flame-induced turbulence, $\Omega$ decreases within the flame in case $\mathrm{C}$ and the peak mean value of $V$ is obtained at the unburned gas (i.e., $c_{T} \approx 0.0$ ) side of the flame front.

\section{CONCLUSION}

The flow topology distributions in different regimes of premixed turbulent combustion were investigated using DNS data of statistically planar turbulent $\mathrm{H}_{2}$-air flames with $\phi=0.7$. The flow topologies were characterized in terms of the first, second, and third invariants (i.e., $P=-\nabla \cdot \vec{u}$, $Q$, and $R$ ) of the velocity gradient tensor $\partial u_{i} / \partial x_{j}$, where invariants $Q$ and $R$ are closely linked with vorticity and strain rates and also their generation rates. The mean value of $\nabla \cdot \vec{u}=-P$ decreases considerably in the broken reaction zone regime due to the severe disruption in chemical processes. The regime of combustion, especially the weakening of dilatation rate effects with increasing Karlovitz number, significantly affects the statistical behaviors of dilatation rate, enstrophy, and strain rate magnitude in addition to the generation rates of enstrophy and dissipation rate. These influences are reflected in the statistical behaviors of the second and third invariants (i.e., $Q$ and $R$ ) of the velocity gradient tensor and their behavior across the flame. 
Detailed explanations were provided for the observed combustion regime dependences of $P$, $Q$, and $R$, the flow topology distribution, and their influences on the scalar-turbulence interaction and vortex-stretching terms in the SDR and enstrophy transport equations, respectively. It was demonstrated that the influences of the combustion regime on $P, Q$, and $R$ have important consequences on the distribution of those flow topologies that make dominant contributions to the scalar-turbulence interaction and vortex-stretching terms in the SDR and enstrophy transport equations, respectively. This analysis identified the flow topologies that make dominant contributions to $\Lambda$ and $V$ and thus the flow configurations responsible for the observed trends in different combustion regimes. This information can help in designing simplified experimental configurations for analyzing the statistical behaviors of the scalar-turbulence interaction and vortex-stretching terms.

\section{ACKNOWLEDGMENTS}

N.C. and D.H.W. are grateful to EPSRC and N8/ARCHER. P.G.A. and H.G.I. were sponsored by KAUST and made use of the resources of the KAUST Supercomputing Laboratory and computer clusters.

[1] A. Perry and M. Chong, A description of eddying motions and flow patterns using critical-point concepts, Annu. Rev. Fluid Mech. 19, 125 (1987).

[2] M. Chong, A. Perry, and B. Cantwell, A general classification of three-dimensional flow fields, Phys. Fluids 2, 765 (1990).

[3] J. Soria, R. Sondergaard, B. Cantwell, M. Chong, and A. Perry, A study of the fine-scale motions of incompressible time-developing mixing layers, Phys. Fluids 6, 871 (1994).

[4] M. Chong, A. Perry, J. Chacin, and B. Cantwell, Turbulence structures of wall-bounded shear flows found using DNS data, J. Fluid Mech. 357, 225 (1998).

[5] A. Ooi, J. Martín, J. Soria, and M. Chong, A study of the evolution and characteristics of the invariants of the velocity gradient tensor in isotropic turbulence, J. Fluid Mech. 381, 141 (1999).

[6] J. Chacin and B. Cantwell, Dynamics of a low Reynolds number turbulent boundary layer, J. Fluid Mech. 404, 87 (2000).

[7] C. Dopazo, J. Martín, and J. Hierro, Local geometry of isoscalar surfaces, Phys. Rev. E 76, 056316 (2007).

[8] E. Elsinga and I. Marusic, Universal aspects of small-scale motions in turbulence, J. Fluid Mech. 662, 514 (2010).

[9] J. Chen, B. Cantwell, and N. Mansour, Proceedings of the Tenth Australasian Fluid Mechanics Conference, Melbourne (Australian Fluid Mechanics Society, 1989).

[10] R. Sondergaard, J. Chen, J. Soria, and B. Cantwell, Proceedings of the Eighth Symposium on Turbulent Shear Flows, Munich (Technical University Munich, 1991).

[11] H. Maekawa, T. Hiyama, and Y. Matsuo, Study of the geometry of flow patterns in compressible isotropic turbulence, JSME Int. J. 42, 846 (1999).

[12] S. Suman and S. Girimaji, Velocity gradient invariants and local flow field topology in compressible turbulence, J. Turbul. 11, N2 (2010).

[13] M. Tanahashi, M. Fujimura, and T. Miyauchi, Coherent fine-scale eddies in turbulent premixed flames, Proc. Combust. Inst. 28, 529 (2000).

[14] R. Grout, A. Gruber, C. Yoo, and J. H. Chen, Direct numerical simulation of flame stabilization downstream of a transverse fuel jet in cross-flow, Proc. Combust. Inst. 33, 1629 (2011).

[15] L. Cifuentes, C. Dopazo, J. Martin, and C. Jimenez, Local flow topologies and scalar structures in a turbulent premixed flame, Phys. Fluids 26, 065108 (2014).

[16] L. Cifuentes, Local flow topologies and scalar structures in turbulent combustion, Ph.D. thesis, University of Zaragoza, 2015.

[17] N. Chakraborty and N. Swaminathan, Influence of the Damköhler number on turbulence-scalar interaction in premixed flames. I. Physical insight, Phys. Fluids 19, 045103 (2007). 
[18] N. Chakraborty, Statistics of vorticity alignment with local strain rates in turbulent premixed flames, Eur. J. Mech. Fluids B 46, 201 (2014).

[19] H. G. Im, P. G. Arias, S. Chaudhuri, and H. A. Uranakara, Direct numerical simulations of statistically stationary turbulent premixed flames, Combust. Sci. Technol. 188, 1182 (2016).

[20] M. P. Burke, M. Chaos, Y. Ju, F. L. Dryer, and S. J. Klippenstein, Comprehensive $\mathrm{H}_{2} / \mathrm{O}_{2}$ kinetic model for high-pressure combustion, Int. J. Chem. Kinet. 44, 444 (2012).

[21] N. Chakraborty, E. R. Hawkes, J. H. Chen, and R. S. Cant, The effects of strain rate and curvature on surface density function transport in turbulent premixed methane-air and hydrogen-air flames: A comparative study, Combust. Flame 154, 259 (2008).

[22] R. J. Kee, J. F. Grcar, M. D. Smooke, and J. A. Miller, A FORTRAN program for modeling steady one-dimensional premixed flames, Sandia National Laboratories Report No. SAND85-8240, 1985.

[23] R. S. Rogallo, Numerical experiments in homogeneous turbulence, NASA Ames Research Center Report No. 81315, 1981.

[24] T. Passot and A. Pouquet, Numerical simulation of compressible homogeneous flows in the turbulent regime, J. Fluid Mech. 181, 441 (1987).

[25] C. S. Yoo, Y. Wang, A. Trouve, and H. G. Im, Characteristic boundary conditions for direct simulations of turbulent counterflow flames, Combust. Theor. Model. 9, 617 (2005).

[26] N. Peters, Turbulent Combustion (Cambridge University Press, Cambridge, 2000).

[27] I. Han and K. Y. Huh, Roles of displacement speed on evolution of flame surface density for different turbulent intensities and Lewis numbers in turbulent premixed combustion, Combust. Flame 152, 194 (2008).

[28] H. Reddy and J. Abraham, Two-dimensional direct numerical simulation evaluation of the flame surface density model for flames developing from an ignition kernel in lean methane/air mixtures under engine conditions, Phys. Fluids 24, 105108 (2012).

[29] C. Pera, S. Chevillard, and R. Reveillon, Effects of residual burnt gas heterogeneity on early flame propagation and on cyclic variability in spark-ignited engines, Combust. Flame 160, 1020 (2013).

[30] Z. M. Nikolaou and N. Swaminathan, Heat release rate markers for premixed combustion, Combust. Flame 161, 3073 (2014).

[31] A. J. Aspden, M. S. Day, and J. B. Bell, Turbulence-flame interactions in lean premixed hydrogen: transition to the distributed burning regime, J. Fluid Mech. 680, 287 (2011).

[32] P. E. Hamlington, A. Y. Poludnenko, and E. S. Oran, Interactions between turbulence and flames in premixed reacting flows, Phys. Fluids 23, 125111 (2011).

[33] H. Carlsson, R. Yu, and X. S. Bai, Direct numerical simulation of lean premixed $\mathrm{CH}_{4} /$ air and $\mathrm{H}_{2}$ /air flames at high Karlovitz numbers, Int. J. Hydrogen Energy 39, 20216 (2014).

[34] N. Chakraborty, M. Champion, A. Mura, and N. Swaminathan, in Turbulent Premixed Flame, 1st ed., edited by N. Swaminathan and K. N. C. Bray (Cambridge University Press, Cambridge, 2011), pp. 76-102.

[35] A. Tsinober, L. Shtilman, and H. Vaisburd, A study of vortex stretching and enstrophy generation in numerical and laboratory turbulence, Fluid Dyn. Res. 21, 477 (1997).

[36] N. Chakraborty and N. Swaminathan, Influence of the Damköhler number on turbulence-scalar interaction in premixed flames. II. Model development, Phys. Fluids 19, 045104 (2007).

[37] S. H. Kim and H. Pitsch, Scalar gradient and small-scale structure in turbulent premixed combustion, Phys. Fluids 19, 115107 (2007).

[38] N. Chakraborty, M. Klein, and N. Swaminathan, Effects of Lewis number on reactive scalar gradient alignment with local strain rate in turbulent premixed flames, Proc. Combust. Inst. 32, 1409 (2009).

[39] S. P. Malkeson and N. Chakraborty, Alignment statistics of active and passive scalar gradients in turbulent stratified flames, Phys. Rev. E 83, 046308 (2011). 